\title{
Positive Airway Pressure Therapy in Sleep-Disordered Breathing
}

\author{
Janna R. Raphelson ${ }^{1}$ (D) $\cdot$ Kimberly Y. Kreitinger ${ }^{1} \cdot$ Atul Malhotra $^{1}$ \\ Accepted: 11 November 2020 / Published online: 23 November 2020 \\ (C) The American Society for Experimental NeuroTherapeutics, Inc. 2020
}

\begin{abstract}
Obstructive sleep apnea (OSA) is a highly prevalent condition with major neurocognitive and cardiovascular health effects. Positive airway pressure (PAP) therapy prevents the collapse of the pharyngeal airway to improve hypoxemia, hypercapnia, and sleep fragmentation caused by OSA. While adherence to PAP therapy has been thought to be a barrier to use, consistent usage is likely much higher than commonly thought. In addition, many strategies have been developed to assist providers in improving their patients' PAP adherence.
\end{abstract}

Key Words Sleep-disordered breathing $\cdot$ obstructive sleep apnea $\cdot$ positive airway pressure therapy.

\section{Introduction}

Obstructive sleep apnea (OSA) is a highly prevalent condition with major neurocognitive and cardiovascular sequela [1]. The disease is characterized by repetitive collapse of the pharyngeal airway resulting in hypoxemia and hypercapnia with associated catecholamine surges [2]. The breathing pattern leads to fragmented sleep resulting in daytime fatigue and impaired executive function. In addition, recurrent catecholamine surges with associated hypoxemia can contribute to cardiovascular and cerebrovascular risk. OSA has been associated with risk of stroke [3, 4] and may be a risk factor for Alzheimer's disease based on recent studies [5]. Thus, recognition and treatment of this condition is imperative. This review will address updates in OSA prevalence estimation, therapeutic interventions, adherence strategies, and potential benefits of continuous positive airway pressure (CPAP) therapy in neurologic disease.

The current treatment of choice for OSA is CPAP therapy. CPAP works by providing a pneumatic splint to the pharyngeal airway maintaining a positive transmural pressure and preventing repetitive pharyngeal collapse [6]. CPAP is typically applied through a nasal or oro-nasal mask and, using modern technology, is reasonably well tolerated by many patients [7, 8]. With CPAP use, gas exchange impairments

\section{Invited Review: Sleep}

Janna R. Raphelson

Jraphelson@health.ucsd.edu

1 Department of Medicine, UC San Diego, San Diego, CA, USA leading to hypercapnia and hypoxemia can be avoided, and sleep fragmentation as well as catecholamine surges are markedly attenuated. Although CPAP adherence is a common concern, many strategies have been developed to improve compliance in ways that are actionable for providers.

\section{How Prevalent Is OSA Worldwide?}

A classic study by Young et al. in NEJM 1993 showed a prevalence of symptomatic OSA of roughly $4 \%$ in men and $2 \%$ in women [9]. This analysis used data from the Wisconsin Sleep Cohort Study, a prospective, longitudinal study of Wisconsin state employees. However, considerable data suggest that these historical estimates may no longer be accurate. Peppard et al. noted a prevalence of OSA of roughly $13 \%$ in men and $6 \%$ in women in the same cohort after roughly 20 years had elapsed [10]. The reason for this increase over time is likely multifactorial but probably influenced by the obesity epidemic, the aging of the population, and improvements in diagnostic technology [11]. Heinzer et al. did a community-based estimate of OSA prevalence in Switzerland and showed almost $50 \%$ of men and $23 \%$ of women had clinically important OSA based on an apnea-hypopnea index $\geq$ $15 / \mathrm{h}$ [12]. In this community-based cohort, the diagnosis of OSA was importantly associated with hypertension, diabetes, and depression suggesting a major impact of this condition even in patients who do not typically come to clinical fruition. These data led to literature-based analysis of the global prevalence of OSA. The estimates varied widely depending on the definitions, and data were not available for the majority of 


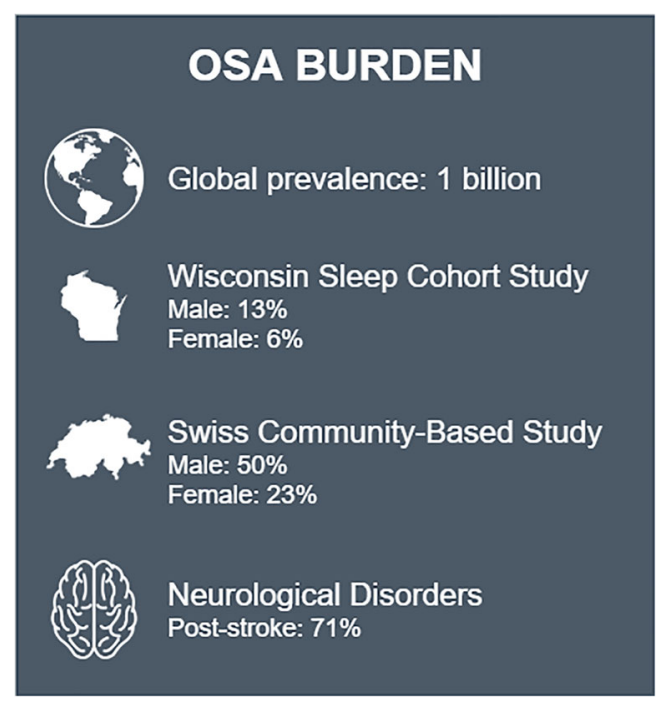

Fig. 1 Studies estimating OSA burden

countries in the world. Nonetheless, the authors estimated up to 1 billion people affected globally with almost 0.5 billion having moderate to severe disease (Fig. 1) [1]. These data speak to the need for a global strategy to address this major burden of disease using approaches that are scalable and portable.

\section{How Can CPAP Therapy Adherence Be Optimized?}

Regarding CPAP adherence, several points deserve emphasis. While some authors and clinicians have suggested that PAP adherence is very poor, the data suggest that adherence to PAP is comparable if not superior to other chronic medical therapies. Using big data analyses, we have recently reported up to $75 \%$ PAP adherence based on Medicare criteria ( $>4 \mathrm{~h}$ night for $70 \%$ of nights) for 2.62 million patients using PAP therapy with an average of $5.1 \mathrm{~h}$ per night in the first 90 days of therapy [8]. Using modern technology, we have reported a propensitymatched analysis whereby patients who received patient engagement software were compared to those who received usual care. The patients with usual care had roughly $70 \%$ adherence with PAP therapy compared to $87 \%$ in those who received active patient engagement with modern technology [7]. As such, a defeatist attitude about CPAP does not seem to be justified. Nonetheless, there is general agreement that new therapeutic strategies need to be developed and that efforts to optimize adherence still have room for improvement (Fig. 2). A number of strategies have been developed to promote CPAP adherence

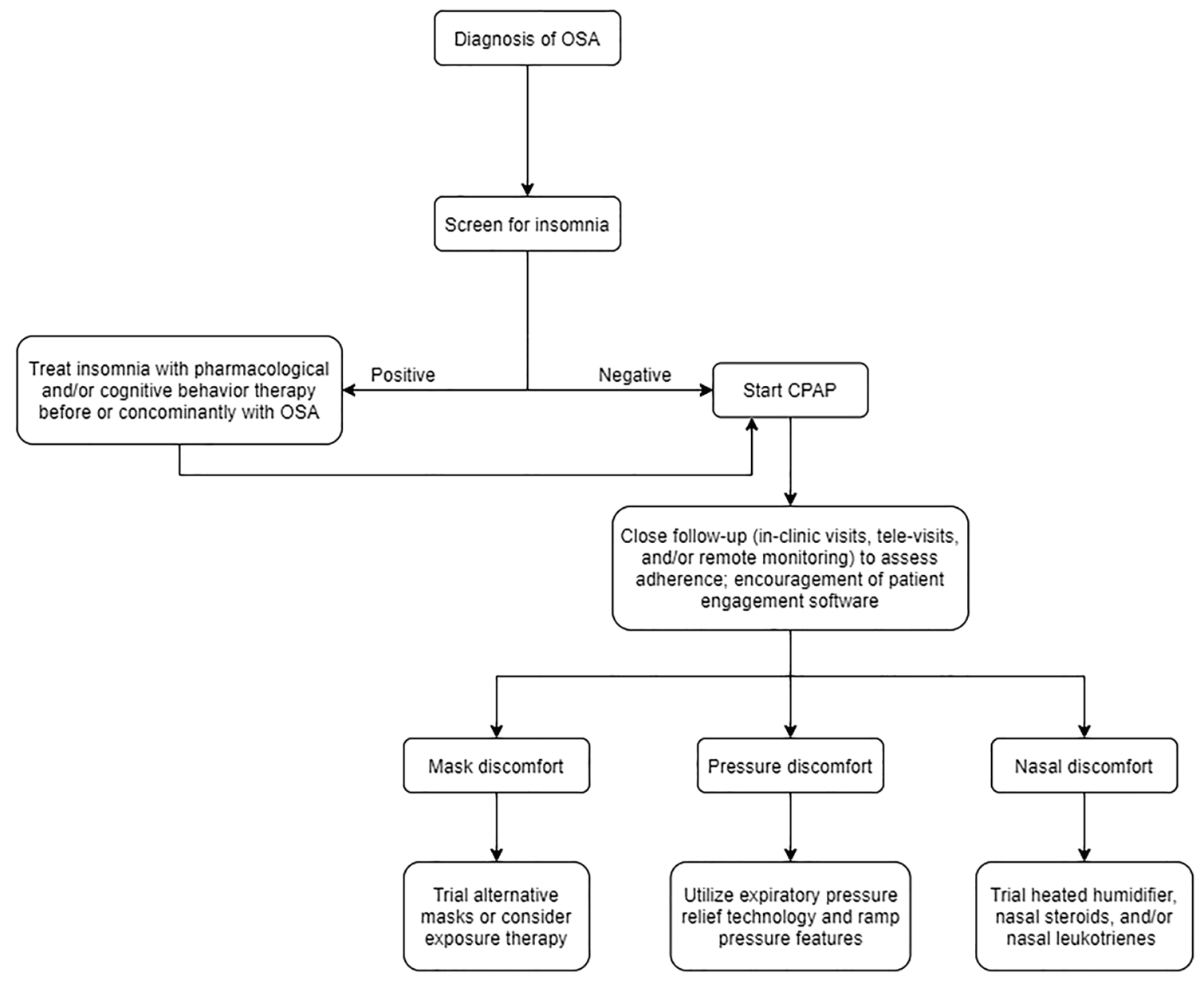

Fig. 2 Management of OSA with CPAP therapy 
including intensive support strategy, tele-education and monitoring, adjunctive pharmacotherapies, and CPAP device modifications [13, 14].

\section{Intensive Support Strategy}

Intensive support is a strategy for improving CPAP adherence that was reported from a randomized controlled trial by Hoy et al. [14]. In this study, all patients were educated on the benefits of CPAP therapy by a sleep physician, sleep nurse, and educational video; received one night of inpatient CPAP titration; and were followed by telephone visits at scheduled intervals during the first month of therapy and by office visits at 1,3 , and 6 months. A randomized cohort of patients also received early education in their home with partner involvement, underwent three nights of inpatient CPAP titration, and were followed up in their homes at 1 week, 2 weeks, 1 month, and 4 months after therapy initiation. The intensive support strategy improved symptom burdens, mood, and cognitive function. Underlying these findings was the higher rates of CPAP usage in the intensive support group with an average of $5.4 \mathrm{~h}$ per night compared to $3.8 \mathrm{~h}$ per night in the control group ( $p=$ 0.003). Although the benefits of intensive support have been variable in the literature, this study should communicate to providers that increased support of CPAP users and partner involvement at the early stage of therapy can lead to robust outcomes. Although support is clearly important, the role of technology to provide this kind of intensive support, especially when compared to traditional clinic visits, remains unclear.

\section{Telemedicine}

The emerging field of telemedicine offers exciting possibilities for further improvement in CPAP adherence. The Tele-OSA study published by Hwang et al. [15] investigated the effect of personalized telemedicine feedback and telehealth education on CPAP compliance. The tele-feedback intervention was guided by a collection of CPAP usage information transmitted from PAP devices. Patients received personalized alerts and encouragement if CPAP usage was at goal over a 3-month period. The tele-education intervention was a series of web-based education modules lasting about $15 \mathrm{~min}$ each that covered CPAP use instructions, potential benefits, and equipment care. The personalized tele-feedback intervention led to increased CPAP usage compared with standard of care with an average of $4.4 \mathrm{~h}$ of usage compared to $3.7 \mathrm{~h}$ of usage ( $p=0.0002)$. Of note, the tele-education intervention was not shown to statistically improve adherence in isolation although it did lead to a 1.3 times higher rate of attendance at further home sleep apnea testing classes.
Personalized telehealth interventions can improve compliance and may be an exciting new method of providing support remotely without intensive personnel demands.

\section{Pharmacologic Strategies}

A common theme in CPAP adherence interventions is timing of intervention as early CPAP usage is one of the strongest predictors of continued adherence. It also seems intuitive to offer support to patients early in their clinical course as they form habits around their therapy. Another potential intervention is the use of pharmacotherapy to ease this transition. In a 2009 randomized controlled trial published by Lettieri et al., a 14-day course of eszopiclone during CPAP therapy initiation was shown to improve duration of CPAP use per night and led to lower rates of therapy discontinuation [16]. Eszopiclone is a non-barbiturate hypnotic primarily used in treatment of insomnia that is safe for use in OSA [17]. Patients in this trial were prescribed this medication for the primary purpose of aiding in transition to therapy regardless of underlying insomnia. The findings of this study prompt the question of whether or not patients with comorbid insomnia can receive further benefit from CPAP if their underlying insomnia is addressed, and in fact, a 2019 study by Sweetman et al. showed that nonpharmacologic therapy for insomnia improved CPAP use [18-20]. Cognitive behavioral therapy for insomnia before initiation of CPAP therapy improved nightly CPAP adherence by an average of $61 \min (p=0.023)$. The authors encourage screening for insomnia before CPAP initiation. These studies suggest that promoting sleep during CPAP initiation may have value regardless of the method taken.

\section{Device Comfort Modifications}

Another intervention for CPAP adherence lies in devicespecific adjustments to make CPAP therapy more comfortable and tolerable. Examples of these strategies are heated humidification and topical steroids to address nasal irritation in patients using nasal CPAP. In a 2009 randomized controlled trial by Ryan et al.,125 patients were assigned to either standard CPAP, CPAP with humidification, or CPAP with nasal steroids [20]. While the addition of humidification decreased nasal symptoms, neither of these interventions improved CPAP compliance. These findings suggest that despite the frequency of nasal complaints these symptoms may not be a major barrier to continued CPAP use. In a further effort to make CPAP more tolerable and comfortable, pressure relief technology has been developed; the feature lowers early expiratory pressure. This strategy has had variable effects on adherence, but in some studies, patients did prefer the technology [21]. In a randomized control trial by Mulgrew et al., pressure relief in combination with autotitrating CPAP was preferred by patients over standard CPAP [22]. Interventions on 
CPAP tolerability and comfort have not been shown to demonstrate as clear an improvement in compliance as strategies focused on early support, psychosocial factor modification, and treating comorbid conditions.

\section{How Is OSA Related to Neurologic Disease?}

With regard to patients with neurological disease, some studies have suggested high prevalence of OSA. A 2019 meta-analysis of studies that included 7096 post-stroke patients found $71 \%$ of these patients had some degree of sleep-disordered breathing [23]. This estimate is much higher than the numbers reported for OSA prevalence in the general population. Whether sleep-disordered breathing predisposes to stroke or is a complication of acute stroke is still unclear. One could postulate that stroke leads to centrally mediated sleep-disordered breathing; however, several studies note a low prevalence of central sleep apnea. Several studies suggest central sleep apnea in this population is actually quite rare, including a 2010 metaanalysis of 29 articles by Johnson et al. [24], which found only $7 \%$ of acute stroke and TIA patients had central sleep apnea. Depending on the etiology of the stroke, however, central apneas may be more common with cardioembolic stroke (with impaired LV function) or with atrial fibrillation. Similarly, it could be presumed that bulbar malfunction due to brainstem ischemia leads to OSA; however, no clear evidence suggests that a single stroke location is predictive of sleep apnea development in post-stroke patients. This finding may indicate that sleep apnea in fact predates stroke and that OSA-induced hypoxia makes acute ischemic penumbra more likely to cause clinically relevant ischemia. Regardless of the etiology of post-stroke sleepdisordered breathing, it is important to identify these patients as stroke patients with OSA have been shown to have less stroke-related impairment if PAP therapy is a part of their post-stroke rehabilitation [25].

Studies have linked OSA with structural changes in the brain. Several small studies have shown gray matter alterations, particularly in the hippocampal and parahippocampal areas in those with OSA [26-30]. Data suggest that treatment can reverse some of these changes. Canessa et al. showed increases in gray matter volume in areas of the brain involved with memory and executive function after 3 months of CPAP treatment in 17 patients with severe OSA [30]. Markers of white matter integrity have also been found to be lower in patients with OSA [31]. In a study conducted by Castronovo et al. [32], signs of white matter pathology were reversed with CPAP treatment with longer treatment duration having more of an impact.

In Alzheimer's dementia, similar benefits may be seen when CPAP therapy is instituted. In one randomized controlled trial, Alzheimer's dementia patients treated with CPAP performed better on neuropsychological testing after 3 weeks of therapy compared to controls [33].

Patients with OSA and Parkinson's disease may also benefit from CPAP therapy. A longitudinal prospective cohort study by Meng et al. in Parkinsonism \& Related Disorders [34] suggested that OSA was associated with worse Parkinson's disease severity score and motor functioning. With 12 months of CPAP treatment, patients showed a stabilization of their disease severity scores as well as their TimedUp-and-Go testing indicating a slowing of motor disease progression compared to the cohort that did not complete CPAP treatment (Fig. 3).

\section{Future Directions}

Although CPAP has transformative benefits for some patients, there exists considerable room for improvement for the many patients who are intolerant. We speculate here regarding future directions that may come to fruition in the coming years:

1. Patient engagement techniques have been associated with improved adherence in observational studies, but further work is clearly needed. Randomized trials using personalized interventions to optimize adherence could be designed. Wearable devices and smartphones may be useful in providing real-time feedback including supportive information for those doing well and helpful hints for those
Fig. 3 Potential benefits of CPAP therapy in neurological disease

\section{Benefits of CPAP Therapy in Neurologic Disease}
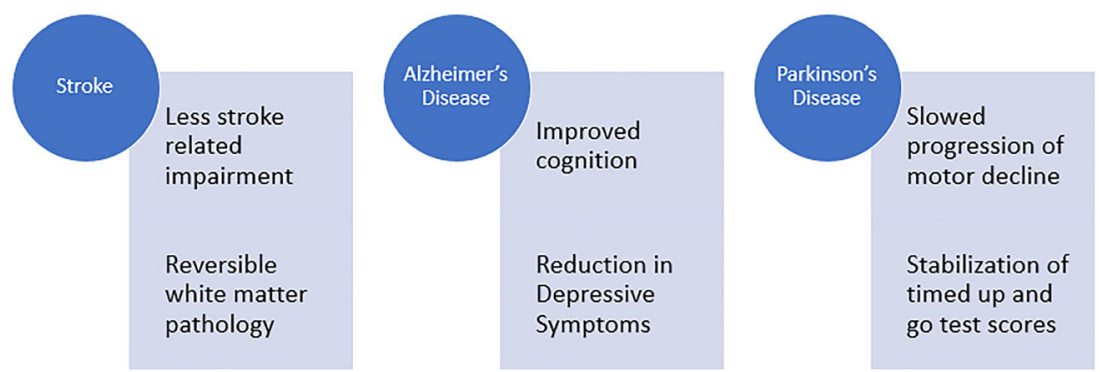
struggling. Such approaches may allow more patients to benefit from CPAP therapy.

2. Pharmacotherapy has been a goal of OSA for many years. Some data support the use of adjunctive sedative/hypnotic agents to facilitate PAP adherence. The use of hypnotic agents has been limited because we currently do not know which patients are likely to benefit. Thus, further work could be undertaken to identify high-risk patients or those most likely to be responsive to adjunctive PAP interventions. Drug therapies are also being investigated for the primary treatment of OSA (e.g., atomoxetine plus oxybutynin as well as dronabinol) [35, 36], but current data are lacking regarding hard outcomes. Nonetheless, future studies could help to develop pharmacological approaches to eliminating apnea at least in a subset of patients.

3. Newer devices are being developed to treat OSA using various approaches. Hypoglossal nerve stimulation has a role in the treatment of OSA at least in select patients. Other approaches to maintaining airway patency are also being investigated including neck suction, neck extension, and various airway splints, but none have robust outcome data. Many are promising and will hopefully provide more targeted upper airway obstruction relief. Previous devices such as a popular brand targeted at relieving local nasal obstruction have had high failure rate overall. In the future, some device strategies may be viable in select patients [37].

In regard to neurologic outcomes in patients with OSA, an exciting new prospective study is underway. The Sleep SMART trial will randomize patients with recent ischemic stroke or transient ischemic attack to therapy with autoadjusting positive airway pressure therapy vs usual care. Primary outcomes will include recurrence of ischemic stroke, ischemic coronary events, all-cause mortality, and standardized recovery scores. Overall, this is a promising study and the results will hopefully add to our knowledge of the interplay between OSA and neurologic outcomes [38].

\section{Conclusion}

OSA is a common condition with a major impact on the overall health of patients including those with neurologic conditions. PAP therapy can provide transformative benefits. Historically, PAP adherence was thought to be variable; however, many strategies have been developed to achieve excellent results in the majority of patients.

Supplementary Information The online version contains supplementary material available at https://doi.org/10.1007/s13311-020-00971-x.
Required Author Forms Disclosure forms provided by the authors are available with the online version of this article.

\section{References}

1. Benjafield AV, Ayas NT, Eastwood PR, et al. Estimation of the global prevalence and burden of obstructive sleep apnoea: a literature-based analysis. Lancet Respir Med 2019;7(8):687-698.

2. Jordan AS, McSharry DG, Malhotra A. Adult obstructive sleep apnoea. Lancet. 2014;383(9918):736-747.

3. Yaggi HK, Concato J, Kernan WN, Lichtman JH, Brass LM, Mohsenin V. Obstructive sleep apnea as a risk factor for stroke and death. N Engl J Med 2005;353(19):2034-2041.

4. Arzt M, Young T, Finn L, Skatrud JB, Bradley TD. Association of sleep-disordered breathing and the occurrence of stroke. Am J Respir Crit Care Med 2005;172(11):1447-1451.

5. Djonlagic I, Guo M, Matteis P, Carusona A, Stickgold R, Malhotra A. Untreated sleep-disordered breathing: links to aging-related decline in sleep-dependent memory consolidation. PLoS One 2014;9(1):e85918.

6. Sullivan CE, Issa FG, Berthon-Jones M, Eves L. Reversal of obstructive sleep apnoea by continuous positive airway pressure applied through the nares. Lancet. 1981;1(8225):862-865.

7. Malhotra A, Crocker ME, Willes L, Kelly C, Lynch S, Benjafield AV. Patient Engagement Using New Technology to Improve Adherence to Positive Airway Pressure Therapy: A Retrospective Analysis. Chest. 2018;153(4):843-850.

8. Cistulli PA, Armitstead J, Pepin JL, et al. Short-term CPAP adherence in obstructive sleep apnea: a big data analysis using real world data. Sleep Med 2019;59:114-116.

9. Young T, Palta M, Dempsey J, Skatrud J, Weber S, Badr S. The occurrence of sleep-disordered breathing among middle-aged adults. N Engl J Med 1993;32:1230-1235.

10. Peppard PE, Young T, Barnet JH, Palta M, Hagen EW, Hla KM. Increased Prevalence of Sleep-Disordered Breathing in Adults. Am J Epidemiol. 2013;177(9):1006-14.

11. McTigue K, Larson JC, Valoski A, et al. Mortality and cardiac and vascular outcomes in extremely obese women. JAMA. 2006;296(1):79-86.

12. Heinzer R, Vat S, Marques-Vidal P, et al. Prevalence of sleepdisordered breathing in the general population: the HypnoLaus study. Lancet Respir Med 2015;3(4):310-318.

13. Hoy CJ, Venelle M, Douglas NJ. Can CPAP use be improved? Am J Respir Crit Care Med 1997;155:304A.

14. Hoy CJ, Vennelle M, Kingshott RN, Engleman HM, Douglas NJ. Can intensive support improve continuous positive airway pressure use in patients with the sleep apnea/hypopnea syndrome? Am J Respir Crit Care Med 1999;159(4 Pt 1):1096-1100.

15. Hwang D, Chang JW, Benjafield AV, et al. Effect of Telemedicine Education and Telemonitoring on Continuous Positive Airway Pressure Adherence. The Tele-OSA Randomized Trial. Am J Respir Crit Care Med 2018;197(1):117-126.

16. Lettieri CJ, Shah AA, Holley AB, Kelly WF, Chang AS, Roop SA. Effects of a short course of eszopiclone on continuous positive airway pressure adherence: a randomized trial. Ann Intern Med 2009;151(10):696-702.

17. Eckert DJ, Owens RL, Kehlmann GB, et al. Eszopiclone increases the respiratory arousal threshold and lowers the apnoea/hypopnoea index in obstructive sleep apnoea patients with a low arousal threshold. Clin Sci (Lond) 2011;120(12):505-514.

18. Sweetman A, Lack L, Bastien C. Co-Morbid Insomnia and Sleep Apnea (COMISA): Prevalence, Consequences, Methodological Considerations, and Recent Randomized Controlled Trials. Brain Sci. 2019;9(12):371. 
19. Sweetman A, Lack L, Lambert S, Gradisar M, Harris J. Does comorbid obstructive sleep apnea impair the effectiveness of cognitive and behavioral therapy for insomnia? Sleep Med 2017;39:38-46.

20. Ryan S, Doherty LS, Nolan GM, McNicholas WT. Effects of heated humidification and topical steroids on compliance, nasal symptoms, and quality of life in patients with obstructive sleep apnea syndrome using nasal continuous positive airway pressure. J Clin Sleep Med 2009;5(5):422-427.

21. Aloia MS, Stanchina M, Arnedt JT, Malhotra A, Millman RP. Treatment adherence and outcomes in flexible vs standard continuous positive airway pressure therapy. Chest. 2005;127(6):20852093.

22. Mulgrew AT, Cheema R, Fleetham J, Ryan CF, Ayas NT. Efficacy and patient satisfaction with autoadjusting CPAP with variable expiratory pressure vs standard CPAP: a two-night randomized crossover trial. Sleep Breath 2007;11(1):31-37.

23. Seiler A, Camilo M, Korostovtseva L, et al. Prevalence of sleepdisordered breathing after stroke and TIA: A meta-analysis. Neurology. 2019;92(7):e648-e654.

24. Johnson KG, Johnson DC. Frequency of sleep apnea in stroke and TIA patients: a meta-analysis. J Clin Sleep Med 2010;6(2):131-137.

25. Ryan CM, Bayley M, Green R, Murray BJ, Bradley TD. Influence of continuous positive airway pressure on outcomes of rehabilitation in stroke patients with obstructive sleep apnea. Stroke. 2011;42(4):1062-1067.

26. Morrell MJ, McRobbie DW, Quest RA, Cummin AR, Ghiassi R, Corfield DR. Changes in brain morphology associated with obstructive sleep apnea. Sleep Med 2003;4(5):451-454.

27. Macey PM, Harper RM. OSA brain morphology differences: magnitude of loss approximates age-related effects. Am J Respir Crit Care Med 2005;172(8):1056-1057; author reply 1057-1058.

28. Macey PM, Henderson LA, Macey KE, et al. Brain morphology associated with obstructive sleep apnea. Am J Respir Crit Care Med 2002;166(10):1382-1387.

29. Yaouhi K, Bertran F, Clochon P, et al. A combined neuropsychological and brain imaging study of obstructive sleep apnea. J Sleep Res 2009;18(1):36-48.
30. Canessa N, Castronovo V, Cappa SF, et al. Obstructive sleep apnea: brain structural changes and neurocognitive function before and after treatment. Am J Respir Crit Care Med 2011;183(10):14191426.

31. Macey PM, Kumar R, Woo MA, Valladares EM, Yan-Go FL, Harper RM. Brain structural changes in obstructive sleep apnea. Sleep. 2008;31(7):967-977.

32. Castronovo V, Scifo P, Castellano A, et al. White matter integrity in obstructive sleep apnea before and after treatment. Sleep. 2014;37(9):1465-1475.

33. Ancoli-Israel S, Palmer BW, Cooke JR, et al. Cognitive effects of treating obstructive sleep apnea in Alzheimer's disease: a randomized controlled study. J Am Geriatr Soc 2008;56(11):2076-2081.

34. Meng L, Benedetti A, Lafontaine AL, et al. Obstructive sleep apnea, CPAP therapy and Parkinson's disease motor function: A longitudinal study. Parkinsonism Relat Disord 2020;70:45-50.

35. Taranto-Montemurro L, Messineo L, Sands SA, et al. The Combination of Atomoxetine and Oxybutynin Greatly Reduces Obstructive Sleep Apnea Severity. A Randomized, Placebo-controlled, Double-Blind Crossover Trial. Am J Respir Crit Care Med 2019;199(10):1267-1276.

36. Carley DW, Prasad B, Reid KJ, Malkani R, Attarian H, Abbott SM, Vern B, Xie H, Yuan C, Zee PC. Pharmacotherapy of Apnea by Cannabimimetic Enhancement, the PACE Clinical Trial: Effects of Dronabinol in Obstructive Sleep Apnea. Sleep. 2018;41(1):zsx184.

37. Friedman M, Hwang MS, Yalamanchali S, Pott T, Sidhu M, Joseph NJ. Provent therapy for obstructive sleep apnea: Impact of nasal obstruction. Laryngoscope. 2016;126(1):254-9.

38. Brown DL, Durkalski V, Durmer JS, Broderick JP, Zahuranec DB, Levine DA, Anderson CS, Bravata DM, Yaggi HK, Morgenstern LB, Moy CS, Chervin RD. Sleep for Stroke Management and Recovery Trial (Sleep SMART): Rationale and methods. Int J Stroke 2020 4:1747493020903979.

Publisher's Note Springer Nature remains neutral with regard to jurisdictional claims in published maps and institutional affiliations. 